\title{
Una experiencia para fomentar los procesos de gestión de la calidad académica en las carreras no acreditadas en la UNED: caso ECEN
}

\author{
Aida B. Azze Pavón \\ Escuela de Ciencias Exactas y Naturales. aazze@uned.ac.cr
}

\begin{abstract}
RESUMEN
La universidad, dada su estructura organizativa actual, no prevé la figura organizativa de carrera (conformada por el programa y las cátedras afines), figura que en el contexto nacional e internacional de la Educación Superior promueve la articulación de la docencia, investigación y la extensión con la gestión de la calidad académica. Se plantea como problema la necesidad de implementar a nivel de carrera, un modelo para la Gestión de la Calidad Académica, que integre las funciones académicas sustantivas: docencia, investigación. El propósito es: "Proponer un modelo de Gestión de la Calidad Académica a nivel de carrera, que facilite el mejoramiento continuo de la calidad académica, acorde a los criterios de calidad nacionales e internacionales para la educación no presencial". La metodología desarrollada, incluye el estudio documental, el análisis de sistemas y sus relaciones, así como la experiencia desarrollada en la aplicación práctica del modelo en las carreras no acreditadas de la Escuela de Ciencias Exactas y Naturales (ECEN). Como resultado de este proceso de estudio y su aplicación práctica, se espera reflexionar en torno a la necesidad de implementar un modelo de Gestión de la Calidad Académica para las carreras de la UNED, acorde a criterios de calidad y estándares reconocidos nacional e internacionalmente para educación no presencial, y sus relaciones con el Círculo de la Calidad como modelo de gestión: planear, ejecutar, documentar, evaluar y tomar nuevas decisiones.
\end{abstract}

\section{ABSTRACT}

The Costa Rican Distance University, given its current organizational structure, does not provide an appropriate organizational structures for careers (made up of programs and related chairs) to fit in the national and international context of higher education that articulates teaching, research and extension to the management of academic quality. Here we propose an Academic Quality Management model to integrate these three substantive academic functions. The methodology includes the analysis of documents, systems and their relationships as well as the experience gained in the practical application of the model in non-accredited careers of the Natural and Exact Sciences School at the Costa Rican Distance University. We hope to obtain conclusions valid for the distance education model and to insert them in the so called Circle of Quality as a management model: plan, execute, document, evaluate and make new decisions.

\section{Introducción}

\section{Antecedentes}

En las mociones del II y III Congreso de la UNED, se denota la tendencia a ir desarrollando procesos de autoevaluación, los procesos de gestión en las escuelas, y más delante de la gestión académica. A continuación se presentan algunas mociones en esta línea.

En el II Congreso de la UNED, se aprobaron las siguientes mociones, entre otras:

Moción 012. Como parte de los procesos de autoevaluación, la UNED someterá a juicio permanente sus discursos y acciones para que:

1. sean coherentes con los principios filosóficos definidos en la misión y en los objetivos.

2. sirvan de base para el quehacer universitario.

Moción 019: Ejercer la autonomía de las escuelas para que ellas mismas determinen su modelo de gestión, planeamiento académico, de la docencia y a evaluación de los procesos de acuerdo con sus especificidades, y generen un desempeño eficiente en la consolidación de sus programas y cátedras. UNED (2009)

Moción 020. Actualizar la descripción de funciones y la carga laboral académica y 
profesional, de manera que incluya las tres ramas del quehacer universitario. Docencia, investigación y extensión.

En el III Congreso. Numeral 4. "Importancia y renovación de la gestión Académica", la moción 15 , en sus puntos 4 y 6 plantea.

Moción 15. Punto 4. Continuar con los procesos de autoevaluación y acreditación de los programas docentes, velando por la elaboración y ejecución de los planes de mejora resultantes.

Punto 6. Garantizar la existencia de planes operativos y su ejecución en las diferentes unidades, coherentes con el Plan de Desarrollo Institucional.

\section{Objetivo del IV Congreso}

El IV Congreso de la UNED, tiene como objetivo general: Innovar la Gestión Académica del modelo educativo de la UNED, de acuerdo con las realidades de la institución y las tendencias del contexto nacional e internacional, mediante el consenso de lineamientos generales que articulen y dinamicen el quehacer académico de la institución. UNED-CU (2011)

Entre los objetivos específicos del Área: Docencia Investigación, extensión, se declara:

Proponer un modelo de Gestión Académica acorde con la realidad actual de la UNED y el contexto nacional e internacional, el cual contemple la inclusión de los ambientes virtuales.

Como respuesta a las mociones del II y III Congreso, en la ECEN se desarrolla una propuesta de Gestión de la Calidad Académica, y su implementación durante el año 2011 en todas las carreras no acreditadas de la Escuela.

\section{Propósito de la ponencia}

Por lo que el propósito de esta ponencia en base a la experiencia desarrollada en la ECEN, es

"Proponer un modelo de Gestión de la Calidad Académica a nivel de carrera, que facilite el mejoramiento continuo de la calidad académica, acorde a los criterios de calidad nacionales e internacionales para la educación no presencial”.

\section{Marco metodológico}

Para la elaboración de perspectiva teórica y el modelo de gestión, los métodos investigativos desarrollados en éste trabajo han sido: El método genético, para determinar la célula en la que confluye lo conceptual, organizativo y humano: la carrera, y lo procedimental objeto de investigación: La Gestión de la Calidad Académica., acorde a criterios y estándares de calidad establecidos nacionalmente y reconocidos internacionalmente. El método dialéctico y sistémico de análisis, para analizar la relación entre los componentes y las propiedades del sistema en movimiento. Métodos que han permitido determinar la relación Círculo de la Calidad vs. Criterios de la Calidad y Estándares, para el desarrollo de la Gestión Académica. El estudio de la información ha conllevado a la utilización de los procedimientos de análisis y síntesis, abstracciones y concreciones, inducciones y deducciones, así como comparaciones, entre otros.

Las fuentes de información utilizadas han sido en primera instancia la revisión documental, tanto bibliográfica como por internet; como complemento y validación se ha utilizado la consulta a encargados de cátedra y programa durante el desarrollo de los talleres en cada grupo-carrera, además de contar con la experiencia adquirida en los procesos de la calidad y autoevaluación de las carreras de la ECEN. Para el desarrollo de los talleres, la estrategia metodológica consistió en analizar los criterios de calidad y estándares del modelo a distancia del Sistema Nacional de Acreditación (SINAES, 2011) y la importancia de la implementación de la gestión de la calidad y los sistemas de información para el logro de los criterios y estándares de calidad académica.

Metodológicamente, en los talleres se fue avanzado por etapas, en las que cada grupo carrera, evidencia las particularidades de sus experiencias e inquietudes para alcanzar en un período de 4 años los criterios de calidad 
SINAES (2011) para una eventual acreditación con el Modelo SINAES.

El proyecto se inició con la carrera Ingeniería Informática, la cual está en proceso de autoevaluación para acreditación, lo que facilitó por su objeto de estudio y condiciones, el diseño de las bases de datos para la digitalización de la información. Continuando con las carreras que han transitado por cambios sustanciales en planes de estudios, con el fin de garantizar el mejoramiento continuo y la validación de los mismos ante la Oficina de Planificación de la Educación Superior: Estas carreras son: Administración de Servicios de Salud, Ingeniería Agroindustrial, Ingeniería Agronómica y Administración de Empresas Agropecuarias, y el programa Diplomado en Registros y estadísticas de Salud, nueva oferta de la ECEN.

\section{Desarrollo}

Se presenta a continuación el marco contextual y el teórico de la ponencia, la que brindará la argumentación necesaria para el análisis de ésta propuesta.

\section{El contexto.}

\section{La Escuela de Ciencias Exactas y Naturales}

La ECEN tiene como razón de ser y como propósito: ser una unidad de formación académica superior sustentada en el modelo de educación a distancia, que desarrolla e integra la docencia, la investigación y la extensión para ofrecer a la sociedad una alternativa de educación de calidad que responda a las demandas de entorno con eficiencia y misión humanística. (ECEN, 2008)

Para el logro de su misión, desarrolla docencia, investigación y extensión para lo cual ha desarrollado equipos de trabajos investigativos y de extensión, a nivel de escuela con el apoyo de los programas y cátedras, política que ha permitido obtener interesantes e innovadores resultados por el equipo de académicos y estudiantes de la escuela, así como extenderlos a la sociedad.
Para promover la integración de las líneas de investigación, de las carreras con la escuela, se crea la Comisión de Investigación y Extensión de la ECEN, la que tiene entre sus objetivos de trabajo: Armonizar las líneas de investigación de la escuela (programas y cátedras), con el fin de integrarlas y oficializarlas dentro de las líneas de la Vicerrectoría de Investigación. Actualmente, esta comisión, ha participado activamente con los programas y cátedras afines en la determinación de las líneas de investigación de la ECEN, con el fin de crear las bases para la articulación de la docencia, investigación y extensión.

\section{Las lecciones aprendidas de \\ los procesos de acreditación}

Los procesos de acreditación de la calidad de las carreras de la ECEN, han dejado en evidencias algunas debilidades en proceso de superación en las carreras acreditadas, reacreditada o en proceso de acreditación ante SINAES. Las principales causas que sustentan las debilidades planteadas por los pares, han sido definidas en el documento (Azze, 2009). Literalmente así planteadas:

- La actual forma de interactuar (estructura organizacional-funcional) del programa y las cátedras que le ofrecen servicios, no favorece el desarrollo con calidad (en particular la actualidad, eficiencia, eficacia, impacto) de las "carreras" que oferta la UNED.

- La poca o casi ninguna identificación y relación de las áreas académicas, personal académico con los estudiantes que estudian "carrera".

- La inexistencia de una cultura de gestión de la calidad académica y del cambio, en programa y cátedras, apoyada por la aplicación continua del Círculo de la Calidad": planificar, ejecutar/evaluar, documentar, tomar decisiones, lo que fomentaría la toma de decisiones para el mejorar los procesos de formación de profesionales. 
En este mismo documento, se expresa como recomendaciones:

- Constituir equipos académicos de carrera, modificando la forma de interactuar (estructura organizacional-funcional) de programas y cátedras en el marco de la escuela y en relación con otras escuelas, con el objetivo de favorecer la calidad de las carreras que ofrece la ECEN y la UNED,

- Implementar la gestión de la calidad y el cambio, apoyada por la aplicación continua del Círculo de la Calidad": planificar, ejecutar/evaluar, documentar, tomar decisiones, en todos los procesos académicos, para lograr el cambio a nivel de Carrera.

- Establecer sistemas de información que permitan conocer a los estudiantes de carrera, como base para la integración y relación de las áreas académicas, personal académico con la Carrera y del personal académico con los estudiantes.

\section{El Compromiso por la calidad de la ECEN}

La Escuela de Ciencias Exactas y Naturales, comprometida con la gestión de la Calidad, ha garantizado a partir del año 2010 la existencia de espacios físicos propios para cada "equipocarrera", aspecto que influye en la pertinencia al grupo, a la socialización e integración de las actividades a desarrollar en torno al proceso de gestión.

Las lecciones aprendidas referidas a la gestión de la calidad académica, por la ECEN en los procesos de acreditación, de las carreras Enseñanza de la Matemática, Enseñanza de las Ciencias Naturales, y Manejo de Recursos Naturales.

Las mociones planteadas en el 2do y 3er Congreso de la UNED, congruentes con la necesidad de garantizar la Gestión de la Calidad Académica se plantea como objetivo de trabajo de la ECEN, (POA, 2011. Punto 4.111.1):

"Implementar la Gestión de la Calidad, en las Carreras que se ofertan en la Escuela, con el fin de elevar la calidad de los procesos académicos que en estas se desarrollan".

\section{Perspectiva teórica}

Se tuvo en cuenta para la elaboración del modelo y el desarrollo de los talleres, los siguientes conceptos y modelos ya existentes:

1. El Concepto de Carrera. Como célula del trabajo (equipo-carrera), que integra la Gestión de la Calidad Académica y es desarrollada por el personal académico, en su papel de promotor activo del desarrollo integrado de la docencia, la investigación, la extensión, la capacitación; así como la proyección social mediante la divulgación de los resultados, participación en eventos nacionales e internacionales, la publicación docente y científica compartida con la comunidad académica, estudiantes y graduados de la carrera.

2. La Gestión de la Calidad Académica de la carrera, visto como el desarrollo eficiente y flexible de la academia: docencia, investigación y extensión, a partir de la aplicación del Círculo de la Calidad": planificar, ejecutar/ evaluar, documentar, tomar decisiones, Dr. Knoll, J (2003)

3. El Modelo Oficial de Acreditación de carreras no presenciales del (SINAES).

Al tomar como base para el desarrollo de la Gestión de la Calidad: los Criterios y Estándares de Calidad del SINAES. Agencia aprobada por el Consejo Universitario para la acreditación de las carreras de la UNED. (SINAES, 2011). El Modelo SINAES (2011), está conformado por cuatro "Dimensiones": Contexto, Recursos, Proceso Educativo y Proyección, con un carácter de proceso. Estas dimensiones a su vez están conformadas por componentes, y cada componente cuenta con sus criterios y estándares de calidad. Además de los criterios de admisibilidad de los estudiantes, y de sostenibilidad de la acreditación por la carrera y la institución.

Es de importancia destacar la priorización de este modelo de acreditación en todas sus 


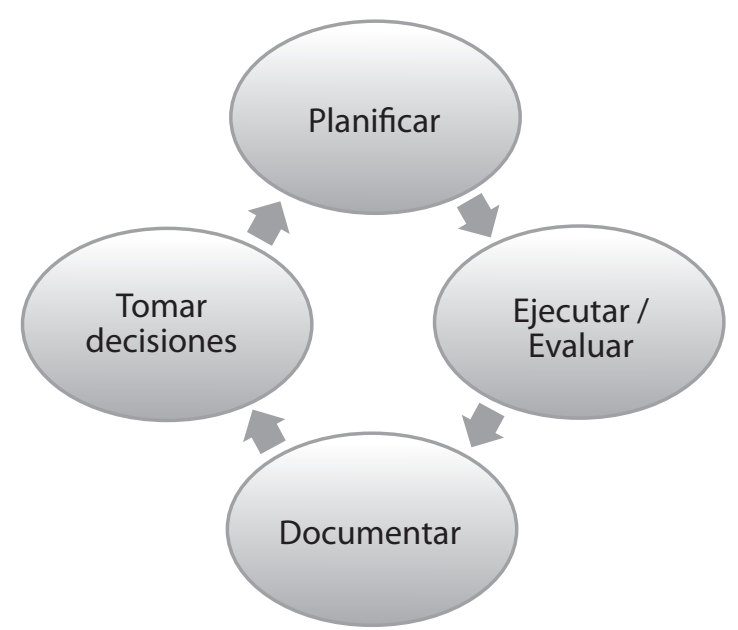

Gráfico 1. Círculo de la Calidad como marco y medio de apoyo de la Gestión Académica. Tomado de Dr. Knoll (2003).

dimensiones de la Integración de la docencia, investigación y extensión. (SINAES, 2011). Lo que se evidencia en particular en los componentes de la Dimensión Proceso Educativo: Desarrollo docente, Investigación, Extensión, Metodología enseñanza aprendizaje, y Vida estudiantil.

4. Relaciones entre el Círculo de la Calidad y las Dimensiones del Modelo SINAES, 2011

Como resultado de la experiencia teóricopráctica que sustenta esta ponencia, se presenta un modelo articulador entre los componentes del Círculo de la Calidad los Criterios y Estándares SINAES (2011) y el concepto de Carrera, los que constituyen una tríada.

Las Dimensiones propias del Modelo SINAES 2011, conforman un proceso continuo de gestión a desarrollar por una carrera, a su vez, en el desarrollo de cada una de las dimensiones están presentes las componentes del Círculo de la Calidad. Ver Gráfico N 3.

Al mismo tiempo existen ejes transversales conformados por los componentes del Círculo de la Calidad que atraviesan diferentes dimensiones para el desarrollo de un mismo componente de la Guía SINAES-2011. Por ejemplo el

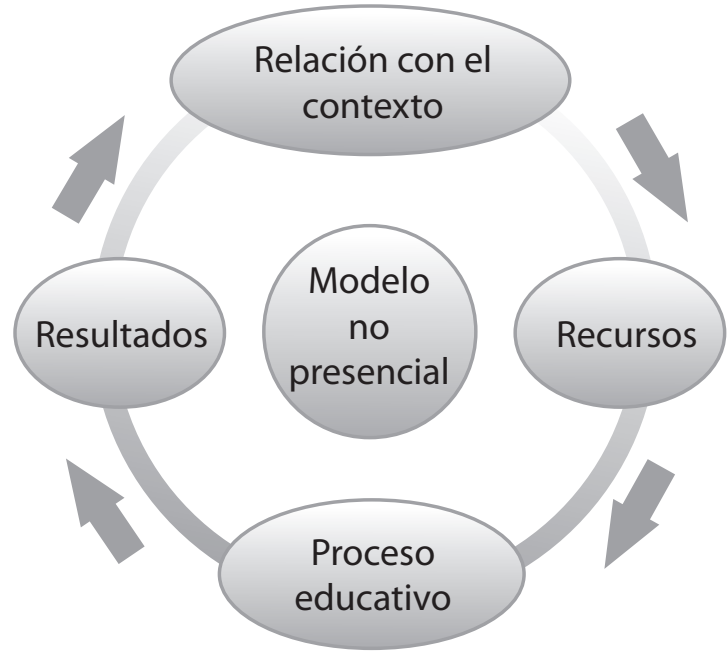

Gráfico 2. Interpretación del Modelo SINAES: Dimensiones.

componente Proceso Docente de la Dimensión Proceso Docente. Ver gráfico 4

Estas relaciones constituyeron la base para la organización de los talleres y de los temas de estudio, teniendo en cuenta la agrupación de los Criterios y Estándares de Calidad de la Guía SINAES (2011) y las acciones que corresponden al cumplimiento del Círculo de la Calidad: Planear, Ejecutar, Documentar, Evaluar y tomar nuevas decisiones. Acciones que garantizan la documentación de la información, el seguimiento, y el mejoramiento continuo.

Los talleres desarrollados, en total cinco, fueron enfocados como proceso, iniciando por el plan y culminando con la toma de decisiones. Los temas tratados en cada taller son:

a. Criterios y estándares de la calidad académica. Vs el Círculo de la Calidad. La sistematización de la información.

b. Gestión del Personal Académico, Investigación, Proyección Social y Desarrollo docente. Criterios y estándares de calidad

c. Gestión del Proceso Docente. Criterios y estándares de calidad

d. Gestión del tránsito de los estudiantes y graduados. Criterios y estándares de calidad 


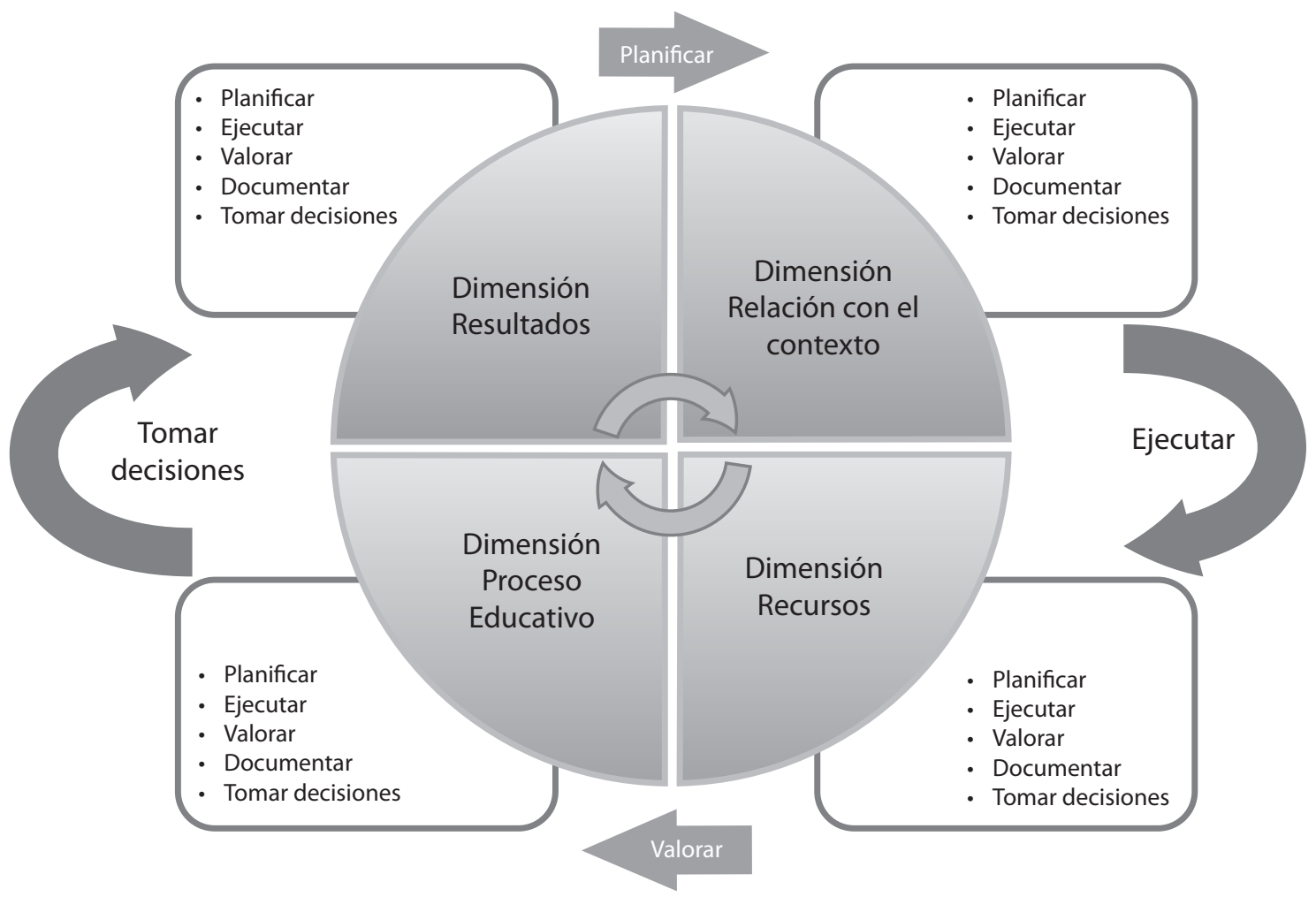

Gráfico 3. Relación entre el Círculo de la Calidad, el Modelo SINAES en una Carrera. Versión A. Azze.

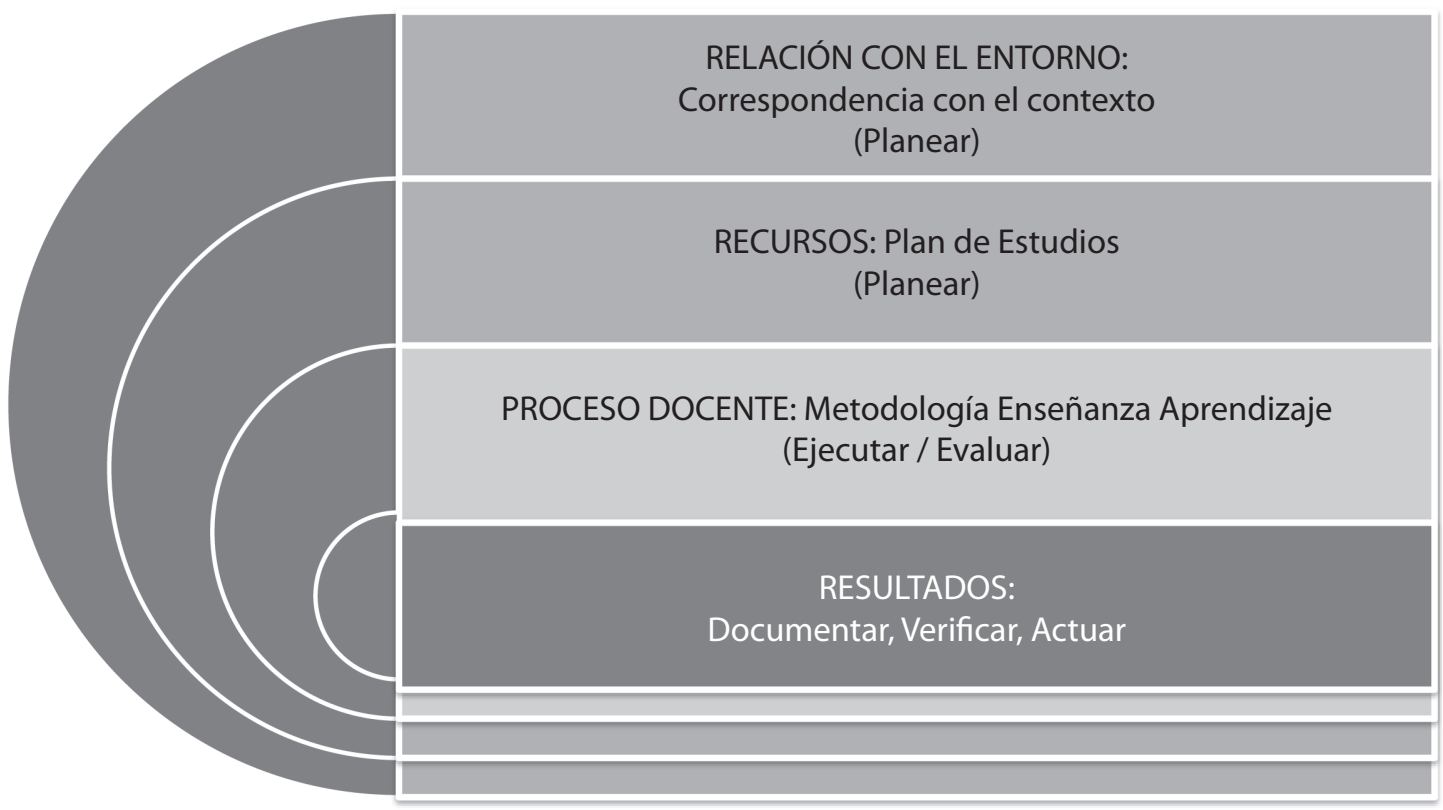

Gráfico 4. Gestión del Proceso Docente. Versión A. Azze. 
e. Gestión de los Recursos Físicos y Materiales. Criterios de calidad y estándares.

5. La conformación de los grupos para la realización de talleres.

La conformación de los grupos facilitó y enriqueció el proyecto, al respetar las culturas propias del equipo-carrera, debido a la diversidad de carreras que se desarrollan en la ECEN,

"Las universidades, facultades y departamentos, hasta las disciplinas científicas mismas tienen sus propias culturas. Y son las culturas las quemuchas veces inconscientemente-deciden sobre las acciones. Las culturas, con sus diferencias, inciden clandestinamente en los detalles de la labor diaria universitaria”. (M. Wesseler 2004)

6. Los sistemas de información.

Para la sistematización y documentación de la Información, se elaboraron de bases de datos digitalizadas de información académica, las que permiten realizar estudios descriptivos y de tendencias de la información requerida para la gestión: personal académico, administrativo, listados de estudiantes y graduados, criterios de admisibilidad de los estudiantes, investigaciones, extensión, publicaciones desarrolladas por las carreras, entre otros. Para lo que se contó con la colaboración de un asesor del programa de Autoevaluación Académica (PAA) y el personal de la carrera Ingeniería en Informática para la elaboración e implementación de los sistemas en cada una de las carreras de la ECEN.

\section{Resultados alcanzados}

Se desarrolla y aplica un modelo de Gestión de la Calidad Académica para ser aplicado en las carreras de la ECEN, que por sus resultados puede ser generalizado a las carreras de la UNED.

Se logró la sensibilización de todos los participantes, generándose un espíritu de grupo al reconocerse en los talleres que el mejoramiento continuo de las carreras es un trabajo de todos.
Se comenzó la digitalización de la información, por los asistentes de los programas, tomándose como modelo de sistema de información para el mejoramiento de la calidad, el que solicita el SINAES para la autoevaluación y acreditación de las carreras con el Modelo no presencial del 2011.

En los talleres participaron, todos los encargados de programa, con las cátedras de sus programas y los asistentes administrativos. La asistencia y la participación en todas las actividades alcanzaron el $100 \%$.

\section{Conclusiones y recomendaciones}

La experiencia fue satisfactoria y permitió iniciar el proceso de Gestión de la Calidad Académica en cada una de las carreras en las que se aplicó, por lo que se recomienda generalizar este tipo de experiencia a las carreras de la UNED, en proceso de mejoramiento, con vistas a una futura acreditación con el SINAES.

Un logro fundamental, es la convicción de los encargados de cátedras y de programa de que el colectivo que ellos conforman constituye una Carrera, y que todos de forma integrada comparten la responsabilidad del desarrollo con calidad del plan de estudios que ofertan, para lo cual deben de trabajar a partir de un mismo propósito en la Gestión de la Calidad Académica.

Considerar la propuesta de Gestión de la Calidad Académica, integrado por la tríada: "Carrera", "Círculo de la Calidad" y los "Criterios de la Calidad y Estándares, definidos por el SINAES (2011)”, como célula del trabajo para el desarrollo con eficacia y eficiencia de la calidad de la oferta académica, gestión que será desarrollada por el personal académico y administrativo del programas y sus cátedras propias con el apoyo de las cátedras de servicio.

\section{Referencias}

Azze. A. B (2009) Debilidades comunes de las carreras autoevaluadas en la ECEN. Niveles de intervención en la UNED. Tomado el 20 de julio del 2011. www.uned. ac.cr/paa/documents/DEBILIDADES_ECEN2009. pdf 
POA-ECEN (2011) Plan Operativo Anual de la Escuela de Ciencias Exactas y Naturales.

Knoll, G. (2003). El Círculo de la Calidad como marco y medio de apoyo. Conferencias del Dr. G. Knoll, en el curso Internacional Unicambio 21. Leipzig. Alemania.

SINAES (2011) Modelo de Acreditación de carreras de grado no presenciales. Documento digital. SINAES. San José. Costa Rica. www.sinaes.ac.cr
UNED (2009) Directrices de la UNED. CIDREB. UNED. Costa Rica.

UNED-CU (2011) Reglamento del IV Congreso Universitario de la UNED.

Wesseler, M. (2004) Apuntes de las conferencias del Dr. Matthias Wesseler en el curso Internacional Unicambio 21. San José. Costa Rica. 